\title{
COINCIDING DIRECTIONS FOR IMBEDDINGS OF SPACES ${ }^{1}$
}

\author{
JAN W. JAWOROWSKI
}

W. T. Wu [4] and [5] and A. Shapiro [3] defined obstruction classes $w^{i}(X)$ to the existence of an imbedding $f: X \rightarrow R^{n}$ of a topological space $X$ in the euclidean $n$-space $R^{n}$. The Wu-Shapiro classes $w^{i}$ are particular cases of the Smith classes defined for a space with an involution. We recall briefly the definition.

1. Equivariant cohomology. Let $A$ be a space with a continuous fixed point free involution $\alpha: A \rightarrow A$. Consider the two possible actions of $\alpha$ on the coefficient group $\boldsymbol{Z}$ of integers: the trivial action and the nontrivial one. Let $H_{+}^{i}(A)$ (resp. $\bar{H}_{+}^{i}(A)$ ) denote the equivariant (resp. residual) $i$ th cohomology group of $A$ for the trivial action; and let $\stackrel{\circ}{H}_{-}^{i}(A)$ (resp. $\bar{H}_{-}^{i}(A)$ ) denote the corresponding equivariant (resp. residual) cohomology groups for the nontrivial action of $\alpha$ on $\boldsymbol{Z}$ (the singular homology is considered throughout; see [1]). The groups $\stackrel{\circ}{H}_{+}^{i}(A)$ and $\stackrel{\circ}{H}_{-}^{i}(A)$ can also be described as the corresponding cohomology groups of the orbit space $A / \alpha$, with the constant and twisted coefficients $\{\boldsymbol{Z}\}$, respectively.

There are exact sequences:

$$
\begin{gathered}
\cdots \rightarrow H^{i-1}(A) \rightarrow \bar{H}_{+}^{i-1}(A) \stackrel{\delta_{i}}{\rightarrow} \stackrel{\circ}{H}_{+}^{i}(A) \rightarrow H^{i}(A) \rightarrow \cdots, \\
\cdots \rightarrow H^{i-1}(A) \rightarrow \bar{H}_{-}^{i-1}(A) \rightarrow \stackrel{\circ}{H}_{-}^{i}(A) \rightarrow H^{i}(A) \rightarrow \cdots
\end{gathered}
$$

Consider the singular chain complex $C(A)$ of $A$ and the chain maps $1+(-1)^{i} \alpha_{i}$, where $\alpha_{i}: C_{i}(A) \rightarrow C_{i}(A)$ is induced by $\alpha$. They induce homomorphisms

$$
\begin{gathered}
\stackrel{i}{i}^{i}: \bar{H}_{-}^{i}(A) \rightarrow \stackrel{\circ}{H}_{+}^{i}(A) \quad \text { if } i \text { is even, } \\
t^{i}: \bar{H}_{+}^{i}(A) \rightarrow \stackrel{\circ}{H}_{-}^{i}(A) \quad \text { if } i \text { is odd. }
\end{gathered}
$$

The homomorphisms $t^{i}$ are known to be isomorphisms (see [5]). Therefore we shall identify the groups $\bar{H}_{-}^{i}(A)$ and $H_{+}^{i}(A)$, if $i$ is even, and the groups $\bar{H}_{+}^{i}(A)$ and $\stackrel{\circ}{H}_{-}^{i}(A)$, if $i$ is odd, under the isomorphisms

Received by the editors January 17, 1967.

${ }^{1}$ Research supported by NSF Grant GP-6015. 
$t^{i}$ and denote them by $\stackrel{\circ}{H}^{i}(A)$. Thus $\stackrel{\circ}{H}^{i}$ denotes $\stackrel{\circ}{H}_{+}^{i}=\bar{H}_{-}^{i}$, if $i$ is even, and $\stackrel{\circ}{H}_{-}^{i}=\bar{H}_{+}^{i}$, if $i$ is odd.

Let $\eta^{p}: \stackrel{\circ}{H}^{0}(A) \rightarrow \stackrel{\circ}{H}^{p}(A)$ denote the homomorphism defined by $\eta^{p}=\delta^{p-1} \circ\left(t^{p-1}\right)^{-1} \circ \cdots \circ \delta^{0} \circ\left(t^{0}\right)^{-1}$.

In particular, $H^{0}(A)=\stackrel{\circ}{+}_{+}^{0}(A)$ and it contains the constant unit cohomology class $1 \in H^{0}(A)$. The cohomology class

$$
s^{p}(A)=\eta^{p}(1) \in \stackrel{\circ}{H}^{p}(A)
$$

is called the $p$ th Smith class of $A$. The index Ind $(A)$ of $A$ is defined to be the maximal in teger $p$ such that $s^{p}(A) \neq 0$ (or $\infty$ if $s^{p}(A) \neq 0$ for all integers $p \geqq 0$ ).

If $A=S^{n}$ and $\alpha: S^{n} \rightarrow S^{n}$ is the antipodal involution, then $H^{i}\left(S^{n}\right)$ $\cong \boldsymbol{Z}_{2}$, for $0<i \leqq n$, generated by $s^{i}\left(S^{n}\right)$; and Ind $\left(S^{n}\right)=n$.

We use a similar notation for homology. Then we have dual isomorphisms

$$
\begin{aligned}
& t_{i}: \stackrel{\circ}{H}_{i}^{-}(A) \rightarrow \bar{H}_{i}^{+}(A) \text { if } i \text { is even, } \\
& t_{i}: \stackrel{\circ}{H}_{i}^{+}(A) \rightarrow \bar{H}_{i}^{-}(A) \text { if } i \text { is odd; }
\end{aligned}
$$

they are induced by the chain map $1+(-1)^{i-1} \alpha_{i}: C_{i}(A) \rightarrow C_{i}(A)$. We again identify the corresponding groups under $t_{i}$ and denote them by $\stackrel{\circ}{H}_{i}(A)$. Thus $\stackrel{\circ}{H}_{i}$ denotes $\stackrel{\circ}{H}_{i}^{-}=\bar{H}_{i}^{+}$, if $i$ is even, and $\stackrel{\circ}{H}_{i}^{+}=\bar{H}_{i}^{-}$, if $i$ is odd.

It is easy to verify that the Kronecker index pairing $\langle\rangle:, \stackrel{\circ}{H}_{i}(A)$ $\otimes \stackrel{\circ}{H}^{i}(A) \rightarrow \boldsymbol{Z}_{2}$ is defined.

If $A$ and $B$ are two spaces with fixed point free involutious and $\phi: A \rightarrow B$ is an involution preserving map, then $\phi^{*} s^{p}(B)=s^{p}(A)$ and thus Ind $(A) \leqq$ Ind $(B)$.

A space $A$ is said to be (-1)-acyclic if it is nonempty; it is said to be $p$-acyclic, $p \geqq 0$, if its cohomology groups $H^{i}(A)$ are isomorphic to those of a one-point space in dimensions $i \leqq p$.

Observe that if $A$ is $p$-acyclic, then the connecting homomorphisms $\delta^{i-1}$ in sequences (1.1) and (1.2) are isomorphisms, for $i \leqq p$, and monomorphisms for $i=p+1$. Thus if $A$ is $p$-acyclic, then Ind $(A)$ $\geqq p+1$.

2. Case mod 2. We obtain a simplified version of the above theory if we use the group $\boldsymbol{Z}_{2}$ as coefficient group. In this case there is only one action of $\alpha$ on the coefficient group, the trivial one, and sequences (1.1) and (1.2) coincide. We denote the resulting homomorphisms analogous to $\eta^{p}$ by 


$$
\stackrel{p}{\eta_{2}}: \stackrel{\circ}{H}^{0}\left(A ; \boldsymbol{Z}_{2}\right) \rightarrow \stackrel{\circ}{H}^{p}\left(A ; \boldsymbol{Z}_{2}\right) ;
$$

the Smith class $\bmod 2$ by $s_{2}^{p}(A)=\eta_{2}^{p}(1)$; and the index $\bmod 2$ by $\operatorname{Ind}_{2}(A)$.

Observe that we have the following commutative diagram (for a nonempty space $A$ ):

$$
\begin{aligned}
& \stackrel{\circ}{H}^{0}(A) \stackrel{\eta^{p}}{\longrightarrow} \stackrel{\circ}{H}^{p}(A)
\end{aligned}
$$

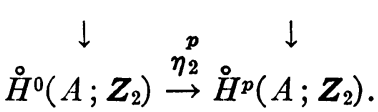

Here the vertical arrows denote the reduction of the coefficients mod 2. Since the homomorphism $H^{0}(A) \rightarrow \stackrel{\circ}{H}^{0}\left(A ; \boldsymbol{Z}_{2}\right)$ carries the unit cohomology class of $\stackrel{\circ}{H}^{0}(A)$ to the unit cohomology class of $\stackrel{\circ}{H}^{0}\left(A ; \boldsymbol{Z}_{2}\right)$, it follows that

$$
\operatorname{Ind}_{2}(A) \leqq \operatorname{Ind}(A) .
$$

REMARK (2.2). It is easily seen that $\operatorname{Ind}_{2}(A)$ coincides with the index of $A$ as defined in [6]. Also, $\operatorname{Ind}_{2}(A) \geqq p$ if and only if $A$ contains a $(p, \alpha)$-system in the sense of [2].

3. Imbeddings into $R^{n}$ and $\mathrm{Wu}$-Shapiro classes. Let $X$ be a space and let $\tilde{X}^{*}$ denote the deleted square $X \times X-\Delta$, where $\Delta$ is the diagonal. Let $\alpha: \tilde{X}^{*} \rightarrow \tilde{X}^{*}$ be the involution permuting the coordinates $\alpha\left(x_{1}, x_{2}\right)=\left(x_{2}, x_{1}\right)$. Then $w^{p}(X)=s^{p}\left(\tilde{X}^{*}\right) \in{\stackrel{\circ}{H^{p}}}^{\left(\tilde{X}^{*}\right)}$ are the Wu-Shapiro classes of $X$. We also denote

$$
I(X)=\operatorname{Ind}\left(\tilde{X}^{*}\right), \quad I_{2}(X)=\operatorname{Ind}_{2}\left(\tilde{X}^{*}\right) .
$$

If $f: X \rightarrow R^{n}$ is a topological imbedding, then the map $\phi: \tilde{X}^{*} \rightarrow S^{n-1}$ defined by

$$
\phi\left(x_{1}, x_{2}\right)=\left(f\left(x_{1}\right)-f\left(x_{2}\right)\right) /\left|f\left(x_{1}\right)-f\left(x_{2}\right)\right|
$$

is involution preserving (for the antipodal involution on $S^{n-1}$ ). Since $S^{n-1}$ is a deformation retract of $\tilde{S}^{n-1 *}$ by a deformation which preserves the involution $\alpha$, it follows that $I(X)<n$. Thus we have

Theorem (3.1) (Wu-Shapiro). If $X$ is imbeddable in $R^{n}$, then $I(X)<n$, i.e. $w^{n}(X)=0$.

This means that $w^{n}(X)$ is an obstruction to the existence of an imbedding $f: X \rightarrow R^{n}$. Wu [5] and Shapiro [3] proved that if $X$ is a finite polyhedron and $n>2$, then the condition $w^{2 n}(X)=0$ is also sufficient for the imbeddability of $X$ in $R^{2 n}$. 
Suppose now that $f: X \rightarrow R^{n}$ and $g: Y \rightarrow R^{n}$ are (topological) imbeddings of spaces $X$ and $Y$ in $R^{n}$. We say that $f$ and $g$ have a coinciding direction if there exist two pairs of distinct points $x_{1}, x_{2} \in X$, $x_{1} \neq x_{2} ; y_{1}, y_{2} \in Y, y_{1} \neq y_{2}$, such that the lines

$$
\overline{f\left(x_{1}\right) f\left(x_{2}\right)} \text { and } \overline{g\left(x_{1}\right) g\left(x_{2}\right)}
$$

are parallel.

The purpose of this paper is to prove the following

THEOREM (3.2). If $I(X)+I_{2}(Y) \geqq n$, then anyimbeddings $f: X \rightarrow R^{n+1}$, $g: Y \rightarrow R^{n+1}$ have a coinciding direction.

Theorem (3.1) can be obtained as a special case of (3.2): if there exists an imbedding $f: X \rightarrow R^{n} \subset R^{n+1}$, let $Y$ be a space consisting of two points, and let $g: Y \rightarrow R^{n+1}$ be an imbedding of $Y$ into a line in $R^{n+1}$ orthogonal to $R^{n}$. Then $f$ and $g$ have no coinciding direction and thus $I(X)+I_{2}(Y)=I(X)<n$.

Actually, a somewhat stronger result than (3.2) will be proved; but the question whether the condition $I(X)+I(Y) \geqq n$ is also sufficient for the existence of a coinciding direction for $f$ and $g$ remains open.

Example (3.3). For $X=Y=S^{1}$, we have $I\left(S^{1}\right)=I_{2}\left(S^{1}\right)=1$. Thus any two simple closed curves in $R^{3}$ have a pair of parallel chords. Similarly, if $Y$ is a triod (a space homeomorphic to the letter $Y$ ), then $I(Y)=I_{2}(Y)=1$. Thus any two triods or a triod and a simple closed curve in $R^{3}$ have coinciding directions.

4. Involution preserving maps into $S^{n}$ and the intersection homomorphism. Let $A$ and $B$ be spaces with involutions $\alpha: A \rightarrow A, \beta: B \rightarrow B$, and let $\phi: A \rightarrow S^{n}, \psi: B \rightarrow S^{n}$ be involution preserving maps (for the antipodal involution of $S^{n}$ ). Let $p$ and $q$ be integers such that $p+q=n$. Then $\phi, \psi$ define a natural intersection homomorphism

$$
\Omega(\phi, \psi): \stackrel{\circ}{H}_{q}(B) \rightarrow \stackrel{\circ}{H}^{p}(A) .
$$

The definition of $\Omega(\phi, \psi)$ can be sketched as follows. Let $T$ be an antipodal cell decomposition of $S^{n}$. For each homology class $\xi_{q} \in \stackrel{\circ}{H}_{q}(B)$, the class $\psi_{*}\left(\xi_{q}\right)$ may be represented by an (equivariant) cycle $z_{q}$ of $T$. The cycle $z_{q}$ defines a homomorphism $u^{p}: C_{p}(A) \rightarrow \boldsymbol{Z}$ as follows: for each chain $c_{p} \in C_{p}(A)$ we can place $z_{q}$ and $c_{p}$ in general position and define $u^{p}\left(c_{p}\right)$ to be the intersection number of $z_{q}$ and $c_{p}$. Since $z_{q}$ is equivariant, the cochain $u^{p}$ is also equivariant and it is actually a cocycle. We define $\Omega(\phi, \psi) \xi_{q}$ to be the cohomology class represented by $u^{p}$. 
By considering two dual antipodal cell decompositions $T$ and $T^{*}$ of $S^{n}$, it can be seen that the (unique) isomorphism $\stackrel{\circ}{H}_{q}\left(S^{n}\right)$ $\cong H^{p}\left(S^{n}\right)\left(\cong \boldsymbol{Z}_{2}\right)$ is just the homomorphism

$$
\omega=\Omega\left(1_{S^{n}}, 1_{S^{n}}\right): \stackrel{\circ}{H}_{q}\left(S^{n}\right) \rightarrow \stackrel{\circ}{H}^{p}\left(S^{n}\right)
$$

which we denote briefly by $\omega ; 1_{S^{n}}$ is the identity map of $S^{n}$; thus $\omega$ is the Poincare isomorphism. It follows that the intersection homomorphism $\Omega(\phi, \psi)$ can be defined equivalently by the following commutative diagram

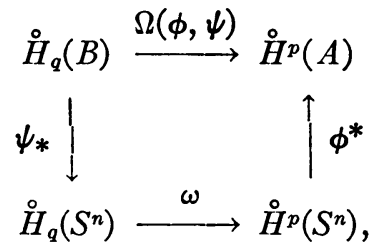

i.e. $\Omega(\phi, \psi)=\phi^{*} \omega \psi_{*}$.

Proposition (4.1). If $A$ and $B$ are spaces with involutions and $\phi: A \rightarrow S^{n}, \quad \psi: B \rightarrow S^{n}$ are involution preserving maps such that $\phi(A) \cap \psi(B)=\varnothing$, then $\Omega(\phi, \psi)=0$.

For $\Omega(\phi, \psi)$ is defined by means of intersection numbers.

Proposition (4.2). If $\phi, \psi$ are as in Proposition (4.1) and $\xi_{q} \in \stackrel{\circ}{H}_{q}(B)$ is a homology class such that the Kronecker index $\left\langle\xi_{q}, s^{q}(B)\right\rangle \neq 0$, then $\Omega(\phi, \psi) \xi_{q}=s^{p}(A)$.

Proof. By the naturality of the Kronecker index and the Smith classes, it follows that $\left\langle\psi_{*}\left(\xi_{q}\right), s^{q}\left(S^{n}\right)\right\rangle \neq 0$; but this means that $\psi_{*}\left(\xi_{q}\right)$ is the unique nonzero element of $\stackrel{\circ}{H}_{q}\left(S^{n}\right)$. Consequently, $\omega \psi_{*}\left(\xi_{q}\right)$ is the unique nonzero element of $H^{p}\left(S^{n}\right)$, i.e. the Smith class $\omega \psi_{*}\left(\xi_{q}\right)$ $=s^{p}\left(S^{n}\right)$. It follows that $\Omega(\phi, \psi)=\phi^{*} \omega \psi_{*}\left(\xi_{q}\right)=s^{p}(A)$.

Due to the fact that there is a unique isomorphism $\iota: \stackrel{\circ}{H}_{q}\left(S^{n} ; Z_{2}\right)$ $\cong H_{q}\left(S^{n}\right)\left(\cong Z_{2}\right)$, we can define an intersection homomorphism

$$
\bar{\Omega}(\phi, \psi)=\phi^{*} \omega \iota \psi_{*}: \stackrel{\circ}{H}_{q}\left(B ; Z_{2}\right) \rightarrow \stackrel{\circ}{H}^{p}(A) .
$$

Propositions corresponding to Propositions (4.1) and (4.2) hold true:

Proposition (4.3). Under the assumptions of Proposition (4.1), $\Omega(\phi, \psi)=0$. 
Proposition (4.4). If $\xi_{q} \in \stackrel{\circ}{H}_{q}\left(B ; \boldsymbol{Z}_{2}\right)$ is a homology class such that the Kronecker index $\left\langle\xi_{q}, s^{q}(B)\right\rangle \neq 0$, then $\bar{\Omega}(\phi, \psi) \xi_{q}=s^{p}(A)$.

For we have again $\left\langle\psi_{*}\left(\xi_{q}\right), s_{2}^{q}\left(S^{n}\right)\right\rangle \neq 0$; it follows that $\psi_{*}\left(\xi_{q}\right)$ is the nonzero element of $\stackrel{\circ}{H}_{q}\left(S^{n} ; Z_{2}\right)$, hence $\iota \psi_{*}$ is the nonzero element of $\stackrel{\circ}{H}_{q}\left(S^{n}\right)$ and $\omega \iota \psi_{*}\left(\xi_{q}\right)=s^{p}\left(S^{n}\right)$. Thus $\bar{\Omega}(\phi, \psi) \xi_{q}=s^{p}(A)$.

TheOREm (4.5). If $A$ and $B$ are spaces with involutions such that $\operatorname{Ind}(A)+\operatorname{Ind}_{2}(B) \geqq n$ and $\phi: A \rightarrow S^{n}, \psi: B \rightarrow S^{n}$ are involution preserving maps, then $\phi(A) \cap \psi(B) \neq \varnothing$.

Proof. There exist integers $p, q$ with $p+q=n$ such that $s^{p}(A) \neq 0$, $s_{2}^{q}(B) \neq 0$. Since $\stackrel{\circ}{H}^{q}\left(B ; \boldsymbol{Z}_{2}\right)=\operatorname{Hom}\left(\stackrel{\circ}{H}_{\boldsymbol{q}}\left(B ; \boldsymbol{Z}_{2}\right), \boldsymbol{Z}_{2}\right)$, there exists a homology class $\xi_{q} \in \stackrel{\circ}{H}_{q}\left(B ; \boldsymbol{Z}_{2}\right)$ such that $\left\langle\xi_{q}, s_{2}^{q}(B)\right\rangle \neq 0$. By virtue of Proposition (4.4), $\bar{\Omega}(\phi, \psi) \xi_{q}=s^{p}(A) \neq 0$; consequently, $\phi(A) \cap \psi(B) \neq \varnothing$, by Proposition (4.3).

Theorem (4.5) implies Theorem (3.2) by letting $A=\tilde{X}^{*}, B=\tilde{Y}^{*}$ and by defining $\phi: A \rightarrow S^{n}, \psi: B \rightarrow S^{n}$ by the formulas

$$
\phi\left(x_{1}, x_{2}\right)=\frac{f\left(x_{1}\right)-f\left(x_{2}\right)}{\left|f\left(x_{1}\right)-f\left(x_{2}\right)\right|}, \quad \psi\left(y_{1}, y_{2}\right)=\frac{g\left(y_{1}\right)-g\left(y_{2}\right)}{\left|g\left(y_{1}\right)-g\left(y_{2}\right)\right|}
$$

for $\left(x_{1}, x_{2}\right) \in \tilde{X}^{*},\left(y_{1}, y_{2}\right) \in \tilde{Y}^{*}$.

In particular, Theorem (4.5) implies that if $A, B$ are two antipodal subsets of $S^{n}$ such that $\operatorname{Ind}(A)+\operatorname{Ind}_{2}(B) \geqq n$, then $A \cap B \neq \varnothing$; here $\phi: A \rightarrow S^{n}$ and $\psi: B \rightarrow S^{n}$ are the inclusion maps. This corollary is stronger than [2, Theorem 2].

\section{REFERENCES}

1. S. Eilenberg, Homology of spaces with operators. I, Trans. Amer. Math. Soc. 61 (1947), 378-417.

2. J. W. Jaworowski, On antipodal sets on the sphere and on continuous involutions, Fund. Math. 43 (1956), 241-254.

3. A. Shapiro, Obstructions to the imbedding of a complex in a euclidean space. I: First obstruction, Ann. of Math. 66 (1957), 256-269.

4. W. T. Wu, On the realization of complexes in euclidean spaces. I, Acta Math. Sinica 5 (1955), 505.

5. - On the realization of complexes in euclidean spaces. I, II, III, Sci. Sinica 7 (1958), 251-297; ibid. 7 (1958), 365-387; ibid. 8 (1959), 133-150.

6. C. T. Yang, On theorems of Borsuk-Ulam, Kakutani-Yamabe-Yujobo and Dyson. I, Ann. of Math. 60 (1954), 262-282.

INDIANA UNIVERSITY 